\title{
Solar Origins of Space Weather and Space Climate: Preface
}

\author{
I. González Hernández • R. Komm • A. Pevtsov • \\ J.W. Leibacher
}

Published online: 21 November 2013

(C) Springer Science+Business Media Dordrecht 2013

As the impact of space weather and climate on daily life is becoming more important, it is timely to discuss the latest research on the solar origin of these phenomena. Recent advances in helioseismology have demonstrated that keys for understanding many aspects of solar activity from flare and CME eruptions to cyclic variations may lie with the subphotospheric plasma dynamics. On the other hand, the advent of synoptic vector magnetic-field measurements opens up a new path to a better understanding of magnetic topology of space-weather source regions on the Sun, e.g. active regions, flares, chromospheric filaments, and CMEs. Furthermore, the space-weather research is rapidly maturing, and is now becoming capable of producing stable space-weather forecasts. Despite this recent progress, many questions remain to be answered, including the future directions for the research and applied components of space weather, the role of ground-based and space-borne instrumentation and networks, and the ways for transitioning the results of research into the operational forecast.

This topical issue is based on the presentations given at the 26th National Solar Observatory (NSO) Summer Workshop Solar Origins of Space Weather and Space Climate held at the National Solar Observatory/Sacramento Peak, New Mexico, USA from 30 April to 4 May 2012. The volume also includes contributions that were not presented at this meeting. More than 60 scientists from the US and abroad came to Sac Peak to exchange their ideas on topics of space weather, to share the results of recent studies, and to discuss the future developments. This unique forum brought together experts in different areas of solar and space physics to help in developing a full picture of the origin of solar phenomena that affect Earth's technological systems on a short and long-term basis. The workshop discussions served as a starting ground for future research, and sparked new collaborations in the field.

Solar Origins of Space Weather and Space Climate

Guest Editors: I. González Hernández, R. Komm, and A. Pevtsov

I. González Hernández $(\varangle)$ · R. Komm · A. Pevtsov · J.W. Leibacher

National Solar Observatory, Tucson, AZ, USA

e-mail: irenegh@nso.edu

J.W. Leibacher

Institut d'Astrophysique Spatial, Orsay, France 
The articles in this volume include theory, model, and observation research on the origin of the solar cycle, solar magnetic features, active-region evolution, eruptive events, as well as a discussion on how to incorporate the research into space-weather forecasting tools.

\section{Acknowledgments}

The 26th NSO workshop was sponsored by the SHINE Program of the US National Science Foundation Division of Atmospheric and Geospace Sciences, the US Air Force Office of Scientific Research, the Solar Physics Division of the American Astronomical Society, and the National Solar Observatory.

\section{Articles}

González Hernández, I., Komm, R., Pevtsov, A., Leibacher, J.W.: 2014, Solar Origins of Space Weather and Space Climate: Preface. Solar Phys. 289, 437. doi:10.1007/s11207-0130454-x.

Nelson, N.J., Brown, B.P., Brun, A.S., Miesch, M.S., Toomre, J.: 2014, Buoyant Magnetic Loops Generated by Global Convective Dynamo Action. Solar Phys. 289, 441. doi:10.1007/ s11207-012-0221-4.

Braun, D.: 2014, Helioseismic Holography of an Artificial Submerged Sound Speed Perturbation and Implications for the Detection of Pre-emergence Signatures of Active Regions. Solar Phys. 289, 459. doi:10.1007/s11207-012-0185-4.

Komm, R., Gosain, S., Pevtsov, A.: 2014, Active Regions with Superpenumbral Whirls and Their Subsurface Kinetic Helicity. Solar Phys. 289, 475. doi:10.1007/s11207-012-0218-z.

Gao, Y., Zhao, J., Zhang, H.: 2014, A Study of Connections Between Solar Flares and Subsurface Flow Fields of Active Regions. Solar Phys. 289, 493. doi:10.1007/s11207-0130274-z.

González Hernández, I., Díaz Alfaro, M., Jain, K., Tobiska, W.K., Braun, D.C., Hill, F., Pérez Hernández, F.: 2014, A Full-Sun Magnetic Index from Helioseismology Inferences. Solar Phys. 289, 503. doi:10.1007/s11207-013-0339-z.

Fontenla, J.M., Landi, E., Snow, M., Woods, T.: 2014, Far- and Extreme-UV Solar Spectral Irradiance and Radiance from Simplified Atmospheric Physical Models. Solar Phys. 289, 515. doi:10.1007/s11207-013-0431-4.

Lefevre, L., Clette, F.: 2014, Survey and Merging of Sunspot Catalogs. Solar Phys. 289, 545. doi:10.1007/s11207-012-0184-5.

Murakőzy, J., Baranyi, T., Ludmány, A.: 2014, Sunspot Group Development in High Temporal Resolution. Solar Phys. 289, 563. doi:10.1007/s11207-013-0416-3.

Gyenge, N., Baranyi, T., Ludmány, A.: 2014, Migration and Extension of Solar Active Longitudinal Zones. Solar Phys. 289, 579. doi:10.1007/s11207-013-0424-3.

Pevtsov, A.A., Bertello, L., Tlatov, A.G., Kilcik, A., Nagovitsyn, Y.A., Cliver, E.W.: 2014, Cyclic and Long-Term Variation of Sunspot Magnetic Fields. Solar Phys. 289, 593. doi:10. 1007/s11207-012-0220-5. 
Panasenco, O., Martin, S.F., Velli, M.: 2014, Apparent Solar Tornado-Like Prominences. Solar Phys. 289, 603. doi:10.1007/s11207-013-0337-1.

Altrock, R.C.: 2013, Forecasting the Maxima of Solar Cycle 24 with Coronal Fe XIV Emission. Solar Phys. 289, 623. doi:10.1007/s11207-012-0216-1.

Yeates, A.R.: 2014, Coronal Magnetic Field Evolution from 1996 to 2012: Continuous Nonpotential Simulations. Solar Phys. 289, 631. doi:10.1007/s11207-013-0301-0.

Choudhary, D.P., Lawrence, J.K., Norris, M., Cadavid, A.C.: 2014, Different Periodicities in the Sunspot Area and the Occurrence of Solar Flares, Coronal Mass Ejections in Solar Cycle 23 - 24. Solar Phys. 289, 649. doi:10.1007/s11207-013-0392-7.

Kahler, S.W., Arge, C.N., Akiyama, S., Gopalswamy, N.: 2014, Do Solar Coronal Holes Affect the Properties of Solar Energetic Particle Events? Solar Phys. 289, 657. doi:10.1007/ s11207-013-0427-0.

Steenburgh, R.A., Biesecker, D.A., Millward, G.H.: 2014, From Predicting Solar Activity to Forecasting Space Weather: Practical Examples of Research-to-Operations and Operationsto-Research. Solar Phys. 289, 675. doi:10.1007/s11207-013-0308-6. 